\title{
ATRIBUCIONES PSICOLÓGICAS, COMPETENCIA Y POSESIÓN CONCEPTUAL DESDE EL EXTERNISMO PSICOLÓGICO
}

\section{PSYCHOLOGICAL ATTRIBUTIONS, COMPETENCE AND CONCEPTUAL POSSESSION FROM A PSYCHOLOGICAL EXTERNALIST PERSPECTIVE}

\author{
Silvia ANDRÉs BALSERA* \\ Universidad Complutense de Madrid
}

Resumen: El objetivo de este artículo es aportar unos criterios para determinar, desde un punto de vista externista, la posesión de un concepto por parte de hablantes semi-competentes. Tomando en consideración un ejemplo de T. Burge, presentaremos la noción de "atribución canceladora» con el objetivo de explicar por qué en ocasiones podemos usar un término general en posición oblicua para especificar las actitudes psicológicas de un individuo aunque éste carezca del concepto expresado por el término relevante.

Palabras clave: externismo; Burge, competencia conceptual; atribuciones psicológicas.

Aвstract: The objective of this paper is to provide some criteria in order to determine conceptual possession in semi-competent speakers from an externalist point of view. Taking into account one of T. Burge's examples, we will present the notion of 'cancelling attribution' with the purpose of explaining why sometimes we can use a general term in an oblique position to specify some psychological attitudes of an individual even though he does not posses the concept expressed by the relevant term.

KEYwORDs: externalism; Burge; conceptual competence; psychological attributions.

*s.andres@filos.ucm.es 
Los partidarios del externismo psicológico sostienen que para individuar los contenidos de las actitudes proposicionales de los individuos hay que tomar en consideración elementos de su entorno externo, de carácter natural o social. En este trabajo pretendo esclarecer la cuestión de qué legitimidad posee un individuo para determinar, desde un enfoque externista, que un hablante posee un determinado concepto, aun cuando las condiciones de determinación del mismo no sean dominadas por él, debido a su carácter externo. Abordaré esta cuestión en dos pasos. En primer lugar, explicitaré una regla que rige las atribuciones conceptuales externistas (la «Máxima del Valor Aparente») y estableceré una comparación entre cuatro casos para extraer una serie de condiciones a partir de las cuales podamos determinar cuándo las proferencias de un hablante permiten el uso de dicha Máxima. En segundo lugar, presentaré la noción de atribución canceladora, una expresión de la que me serviré para distinguir ciertos informes de contenidos psicológicos que, debido a su rol en el proceso de interpretación de las actitudes mentales de los agentes, suspenden la aplicación de la Máxima del Valor Aparente con respecto a las proferencias de un hablante.

\section{Atribuciones externistas de contenidos y la Máxima del Valor Aparente}

El enfoque externista en psicología ha supuesto una remodelación del cuadro desde el cual se establecía la posesión de un concepto y su competencia en el mismo por parte de un individuo (Russell 2010: 190). Aunque el punto principal de esta tesis es defender que el contenido psicológico queda parcialmente determinado por elementos que están «fuera de la cabeza» de los hablantes, la consideración externista de la mente tiene repercusiones más allá de la cuestión de la individuación metafísica de contenidos y pensamientos, comportando una visión renovada de los criterios de atribución/posesión conceptual (Greenberg 2007). En este sentido, los experimentos mentales elaborados para aportar plausibilidad a esta tesis autorizan la atribución de un concepto $C$ a un individuo que no domina por completo las condiciones de aplicación del término «C» pero que, por otro lado, se encuentra en las relaciones (externas) apropiadas con su entorno natural y social. A partir de ello, el externista defiende que el protagonista de los experimentos puede tener pensamientos que involucran el concepto expresado por el término general relevante. Así, aunque las condiciones de aplicación que yo asocio en mi idiolecto con los términos «olmo» y «haya» sean exactamente las mismas («árbol de hoja caduca»), desde un punto de vista externista es posible defender que al usar el término «olmo» expreso el concepto de olmo (no el de 
haya) y tengo pensamientos acerca de estos árboles (y no acerca de las hayas) porque mi entorno social determina la identidad del concepto que figura en mis pensamientos relevantes. Dicha determinación social se produce, según el externismo, porque delego en otros hablantes de mi comunidad lingüística la tarea de conocer las condiciones de aplicación del término «olmo».

\subsection{La Máxima del Valor Aparente como consecuencia del minimalismo externista en la competencia conceptual}

Desde el frente externista, especialmente en su vertiente social ${ }^{1}$, se ha puesto cierto énfasis en destacar como una gran virtud de la individuación externista de nuestros conceptos que nos permita interpretar las palabras de los hablantes en su valor aparente (Ebbs 1996), en sentido de que si Hilary profiere la oración «se me ha estropeado el carburador» el contenido de la creencia que debemos atribuirle es que se le ha estropeado el carburador, un contenido que involucra el concepto carburador, aun cuando dicho hablante es sólo parcialmente competente en el uso de dicho concepto. Según se dice, uno de los efectos de contemplar a los hablantes insertos en una red (externa) de relaciones sociales de deferencia lingüística es tomar en consideración sus palabras como si éstas expresaran aquello que se expresa (en su comunidad) mediante el uso de las mismas. Por mor de la claridad, ofreceré una enunciación de dicha Máxima con respecto a las actitudes proposicionales que son las creencias:

(Máxima del Valor Aparente): Atribuye el contenido de que P en los informes psicológicos destinados a describir las creencias de un hablante que asevera «P» de manera sincera y reflexiva ${ }^{2}$.

1 Especificamos que las observaciones que realizaremos en lo que sigue afectan especialmente al externismo social, entendiendo por tal la tesis que sostiene que los conceptos y contenidos mentales de los hablantes quedan individuados, parcialmente, por las convenciones lingüísticas que los hablantes especialmente competentes de una comunidad de individuos (los «expertos») establecen en relación a los términos usados para expresar esos conceptos y contenidos. Esa variante de externismo es plenamente asimilable a las propuestas anti-individualistas de Burge y también lo es, en parte, en relación a las tesis externistas basadas en las tesis semánticas de Putnam (1975b) —que constituyen la compresión del externismo más extendida - debido a que la hipótesis de la división social del trabajo lingüístico planteada en ese artículo incide en la determinación social del significado de los términos, especialmente de los términos generales de género natural y artificial.

2 Aunque sólo enunciaremos la Máxima del Valor Aparente en relación a las creencias, por ser éstas las actitudes proposicionales de las que nos ocuparemos principalmente en lo 
Esta Máxima establece que el contenido de la creencia que atribuimos a un hablante que profiere asertivamente la oración «P», en tanto que contenido que él ha querido expresar mediante esa proferencia, debe ser idéntico al semánticamente expresado por la oración emitida por él. Ahora bien, parece claro que esta Máxima debería admitir ciertas cualificaciones o, al menos, deberíamos restringir su aplicación ocasional en las tareas de atribución de contenidos, de tal manera que la interpretación a partir del valor aparente de las palabras de un hablante debería realizarse salvo si este hablante es manifiestamente incompetente en el uso de un término ${ }^{3}$. Es más, aunque en muchos casos lo más respetuoso con las intenciones lingüísticas de los hablantes sea interpretar sus proferencias a la luz de dicha Máxima (puesto que entre esas intenciones se encuentra la actitud deferencial hacia los expertos de su comunidad), puede ocurrir que la propia consideración caritativa de los individuos nos prevenga de su uso en un caso concreto, debido a que, por error o ignorancia radicales, tales individuos pueden querer decir mediante sus palabras algo completamente distinto de lo que esas palabras expresan (normativamente) en su lenguaje. Acudiendo a un ejemplo que más adelante examinaremos en detalle, podemos encontrar a un individuo, Mary, que sufre un equívoco lingüístico persistente (no un lapsus), de tal manera que denomina «creencia» al melanoma. En caso de que Mary emitiera la proferencia «no he tenido una sola creencia en mi vida», atentaría contra la racionalidad de este hablante emplear la Máxima del Valor Aparente en su situación e informar acerca de sus pensamientos mediante una atribución como "Mary cree que no ha tenido una sola creencia en su vida». Gracias a ejemplos como éste resulta evidente que la Máxima del Valor Aparente debería estar subordinada al Principio de Caridad, en el sentido de que si atribuir a un individuo pensamientos que involucran el concepto $C$ cuando éste hace uso del término «C» nos lleva a atentar gravemente contra su racionalidad — como ocurre si ese individuo es completamente incompetente el uso de ese término-, entonces la Máxima no debería ser aplicada en su caso ${ }^{4}$.

sucesivo, dicha máxima podría enunciarse, mutatis mutandis, con respecto a otra clase de actitudes (deseos, temores, esperanzas, etc.).

3 Respaldamos enteramente la observación de Nordby (2004: 68), quien afirma que "para que estemos dispuestos a aceptar una interpretación literal, la persona tiene que tener una comprensión mínima [de modo que s]i la palabra es sólo un sonido vacío para esa persona o si su comprensión está completamente cubierta por otra palabra, entonces no deberíamos aceptar una interpretación mental literal», que es justo la clase de interpretación que resulta de la aplicación de la Máxima del Valor Aparente.

${ }^{4}$ Quizá el autor que más ha destacado que el hecho de la individuación externista de nuestros contenidos puede conllevar, en ocasiones, atentar contra el Principio de Caridad es Bilgrami (1992a), (1992b) y (2003). De acuerdo con este autor, el hecho de que el «externis- 


\subsection{Limitaciones de la Máxima del Valor Aparente. Necesidad de indicios de posesión conceptual}

En este punto nos encontramos con el siguiente problema. Aunque los autores externistas han escrito una gran cantidad de trabajos para defender sus tesis (especialmente, para demostrar su compatibilidad con el acceso privilegiado), en ellos se contienen escasas indicaciones positivas (pace Burge 1986b) sobre qué pautas han de seguirse para determinar que un individuo semi-competente en el uso de un término general que realiza usos idiosincrásicos del mismo, posee, no obstante, el concepto expresado por el término relevante. Reparemos, además, en que cuando denunciamos la ausencia (relativa) de dichas pautas lo que estamos indicando es, al mismo tiempo, que carecemos de indicaciones para armonizar la Máxima del Valor Aparente con el Principio de Caridad. Por este motivo, creo necesario trabajar para establecer un límite con respecto a la ignorancia que puede mostrar un hablante sobre el uso convencionalmente asociado a un término general sin que esto impida (a) que este individuo posea el concepto relevante y (b) que la interpretación más caritativa de aquellas de sus proferencias que involucran el término sea la interpretación guiada por la Máxima del Valor Aparente.

Sin embargo, antes indicamos que los autores externistas no nos han dejado completamente privados de textos sobre esta cuestión y, en particular, Burge ha ofrecido una serie de indicaciones al respecto en (1986: 258 y ss.). Así, este autor defiende la tesis, plenamente externista, de que la competencia en un mismo concepto admite grados, en el sentido de que dos individuos pueden poseer un determinado concepto $C$ aunque la competencia que uno y otro tienen en el dominio del mismo difiera. En segundo lugar, en lo que se refiere a la cuestión

mo ortodoxo" — basado en las ideas de Putnam (1975b) y Burge (1979) — legitime la atribución de contenidos sobre la artritis a un individuo que asiente reflexivamente ante la proferencia de oraciones que atribuyen propiedades que, por definición, son falsas de la artritis, constituye el mejor ejemplo de esta clase de atentados. Sin embargo, mi posición en este trabajo es un tanto distinta de la de Bilgrami. En primer lugar, creo que las tesis del «externismo ortodoxo", como él las denomina, son valiosas y esencialmente correctas y, en segundo lugar, considero que esta clase de atribuciones a individuos semi-competentes no sólo no atentan contra su racionalidad, sino que resultan más acordes con sus intenciones lingüísticas (parcialmente deferenciales). El problema que denuncio es, por un lado, que falta cierta definición sobre lo que distingue a un individuo semi-competente en el uso de un término de otro individuo que no es competente en absoluto y, por otro, que el hecho de que puntualmente pueda decirse de un individuo que "cree que P» y que consideremos correcto dicho informe no implica siempre que este individuo posea los conceptos involucrados en el contenido atribuido. 
de cuán básica puede ser la competencia de un hablante en un determinado concepto para poder decir que este individuo posee el concepto en cuestión, Burge afirma que la competencia conceptual mínima de un hablante tiene que ver con su aptitud para «recibir atribuciones en las que el término relevante figura de manera oblicua» y también con su capacidad para «racionalizar mínimamente su uso del término» y «estar en conformidad con la práctica de otros», sin exigir que este hablante sea capaz de proporcionarnos explicaciones normativas del significado del término (Burge 1986: 268).

Ahora bien, sobre estas breves indicaciones cabe señalar lo siguiente. En primer lugar, la idea de que la competencia conceptual se establece en grados me parece perfectamente aceptable, como lo pone de manifiesto la propia plausibilidad del experimento mental en torno al concepto de artritis planteado por Burge, en el cual un individuo semi-competente en ese concepto amerita la posesión del mismo (Burge 1979: 104 y ss.). Bert, como denominaré al protagonista de dicho experimento mental, posee el concepto comunitario de artritis, el mismo concepto que posee el doctor a quien comunica sus temores, a pesar de desconocer en parte las condiciones de aplicación del mismo. Sin embargo, las indicaciones de Burge sobre los requisitos para la posesión conceptual son un tanto insuficientes. Esta insuficiencia tiene que ver con el hecho de que Burge caracteriza las «racionalizaciones responsables» del uso del concepto por parte de un hablante mínimamente competente diciendo que la clase de comprensión que permite efectuar esa clase de racionalizaciones es aquella que se presupone cuando atribuimos a un hablante pensamientos que involucran el concepto relevante. El problema es que esta observación peca de una cierta circularidad, en la medida en que si lo que deseamos averiguar es qué habilidades debe mostrar un individuo en relación a su uso del término "C» para que pueda recibir atribuciones de contenidos que involucran el concepto expresado por ese término, apelar a la intuitiva aptitud para recibir atribuciones de contenidos como una evidencia de que posee el concepto no es algo que nos permita avanzar demasiado. Si lo que deseamos es obtener una aclaración filosófica de las intuiciones que apoyan la atribución de contenidos en situaciones ordinarias, deberíamos examinar qué indicios empíricos e inter-subjetivos se emplean en dichas atribuciones cuando las consideramos intuitivamente plausibles. La primera tarea que pretendo llevar a cabo en el presente trabajo es ofrecer una serie de consideraciones sobre tales indicios, para lo cual cual reservaré la siguiente sección. Para este fin me serviré de una comparación entre el "caso artritis», esto es, el experimento mental en torno al concepto de artritis planteado por Burge en (1979), y otros casos un 
tanto parecidos 5 , prestando especial atención al «caso orangután», basado en un ejemplo que este autor ofrece en ese mismo artículo. Lo más conveniente para avanzar en nuestro propósito es citar la descripción del «caso orangután» por parte de nuestro autor:

Si un hablante generalmente competente y razonable piensa que «orangután" se aplica a una bebida de frutas, seríamos reacios, e incuestionablemente podría inducir a error tomar sus palabras como si revelaran que él piensa que ha estado bebiendo orangutanes para el desayuno en las últimas semanas. Esta incomprensión total con frecuencia parece bloquear la atribución literal de contenido mental, al menos en casos en los que no estamos caracterizando directamente su error. (Contrariamente a la tradición filosófica, no estoy convencido de que este hombre no pueda recibir correcta y literalmente la atribución de la creencia de que un orangután es un tipo de bebida de fruta. Pero no trataré este punto aquí)(Burge 1979: 119-120).

En este ejemplo se plantea la situación de un hablante, a quien llamaremos John, que, por ignorancia o error, denomina «orangután» a un tipo de bebida de fruta (supongamos que es el zumo de naranja). Este autor toma en consideración dos informes sobre atribuciones de contenido a este hablante:

(1) John cree que ha desayunado orangutanes en las últimas semanas

(2) John cree que un orangután es un tipo de bebida de fruta

Con respecto a (1), Burge sostiene que se trata de una atribución que podría llevar a equívocos a un tercero que escucha el informe. Parece intuitivamente claro que el autor está en lo cierto y, además, la impropiedad de esa atribución constituye un buen ejemplo de que, como hemos señalado, en ocasiones debería suspenderse la aplicación de la Máxima del Valor Aparente, ya que usarla en relación a la proferencia de John, quien afirma «he desayunado orangutanes», constituye una flagrante violación del Principio de Caridad.

5 La similitud de estos casos tiene que ver con el hecho de que sus protagonistas realizan, en cada caso, usos idiosincrásicos de un término general. No obstante, tendremos oportunidad de comprobar que existen profundas diferencias entre ellos en relación a su competencia en el concepto expresado por el término relevante. 
Sin embargo, este mismo autor sostiene que en el informe (2) se realiza una atribución "correcta y literal»y, aunque él es consciente de que se trata de una tesis enfrentada a la "tradición filosófica», rehúsa explícitamente entrar en detalles sobre esta cuestión. El segundo objetivo que me propongo en este trabajo es continuar la tarea de Burge y argumentar a favor de que (2) sea una atribución de contenido legítima, aun cuando no lo sean el resto (o la mayoría) de las atribuciones de contenido que, como (1), son realizadas interpretando a la luz de la Máxima del Valor Aparente aquellas de las proferencias de John que involucran el término «orangután». Para lograrlo, propongo contemplar las atribuciones como (2) integradas en un proceso de interpretación de los pensamientos de un individuo. Esto nos llevará a distinguirlas en tanto que desempeńan el rol de «atribuciones canceladoras», es decir, de atribuciones en las que un término («orangután») figura en posición oblicua pero que, en primer lugar, no implican que este individuo posea el concepto comunitario expresado por dicho término y que, en segundo lugar, cancelan la aplicación de la Máxima del Valor Aparente para el resto o la mayoría de las atribuciones en las que figura ese término.

\section{Posesión conceptual en individuos semi-competentes}

Siguiendo con el plan propuesto, dedicaremos esta sección a proponer una serie de indicios que nos permitan determinar cuándo un individuo semi-competente en el uso del término «C» posee el concepto $C$ que se expresa en su comunidad lingüística mediante el uso de ese término. Desde luego, no pretendemos agotar el repertorio de criterios al respecto y cabe pensar que la mayoría de los que ofrecemos gozan de un mayor potencial si son utilizados en sentido negativo, esto es, como síntomas que, de no manifestarse, proporcionarán una buena evidencia de que el sujeto cuya aptitud para recibir la atribución conceptual se discute no posee, finalmente, el concepto relevante ${ }^{6}$. Debe señalarse, en último lugar, que debido a que reconocer la posesión del concepto $C$ supondrá que las proferencias de este individuo que involucran el término "C» han de interpretarse siguiendo la Máxima del Valor Aparente, las pautas que ofreceremos servirán para concretar cuáles son las garantías en la aplicación de dicha Máxima.

\footnotetext{
${ }^{6}$ Además, los casos que plantearemos serán protagonizados por individuos que o bien son claramente semi-competentes o bien son claramente incompetentes en relación a los usos de un término general. Aunque sin duda podrían plantearse casos más dudosos o complicados, nuestro propósito es atender a situaciones en las que los sujetos relevantes presenten de manera nítida los indicios señalados o que, de una manera igualmente obvia, presenten su carencia.
} 


\subsection{Cuatro casos para un ejercicio comparativo}

Con el fin de aportar claridad en nuestro objetivo, tomaremos en consideración una serie de casos protagonizados por individuos que realizan aplicaciones anómalas de un término general:

- El caso artritis, diseñado por el propio Burge, que concierne a un individuo, Bert, que usa el término «artritis» para decir a su médico «temo que la artritis también me está afectando al muslo», aun cuando ignora que dicha enfermedad, por definición, sólo afecta a las articulaciones. ¿Podríamos decir que Bert cree que sufre artritis en el muslo? ¿Podemos aplicar la Máxima del Valor Aparente y atribuir a este individuo la posesión del mismo concepto que expresa su médico mediante el uso del término artritis?

- El caso orangután, recogido en la sección anterior y planteado en torno a un individuo, John, que denomina «orangután» a una bebida de fruta (el zumo de naranja) y que profiere la oración «he desayunado orangutanes en las últimas semanas». En este caso, ¿podemos realizar una atribución literal y decir que John cree que ha desayunado orangutanes en las últimas semanas? ¿Posee este individuo el concepto de orangután?

- El caso silla, basado en la versión de Burge (1986: 259), en el cual un individuo, Max, desconoce que las sillas pueden no tener patas, por lo que se equivoca al rechazar la aplicación del término «silla» a asientos unipersonales que carecen de patas. A pesar de ello, ¿cabe afirmar que Max tiene creencias que involucran el concepto de silla? ¿Posee Max ese concepto?

- El caso creencia, protagonizado por un hablante, Mary, que denomina "creencia» a un tipo de cáncer, en particular, al melanoma y que profiere la oración «no he tenido ninguna creencia en toda mi vida». ¿Podemos atribuir a Mary la peculiar creencia de que no ha tenido ninguna creencia en toda su vida? ¿Posee este individuo el concepto de creencia?

Mediante el planteamiento de estos casos pretendo averiguar si sus protagonistas, que realizan usos idiosincrásicos de los términos «artritis», «orangután», «silla» y "creencia», poseen los conceptos que en su comunidad se expresan haciendo uso de tales términos. Por ahora, no atenderemos a la clase de atribuciones que, en palabras de Burge, "caracterizan el error de los hablantes», atribuciones como "John cree que los orangutanes son un tipo de bebida de fruta" 
o «Mary cree que las creencias son un tipo de cáncer» y que este autor considera "correctas». Por tanto, sólo examinaremos los informes como «Mary cree que la exposición excesiva al sol provoca creencias» o "John cree que los hoteles deberían ofrecer orangutanes naturales en sus desayunos». Según concluiremos, sólo Bert (caso artritis) y Max (caso silla) serán hablantes semi-competentes que poseen el concepto comunitario aunque desconozcan en parte las condiciones de aplicación del mismo.

\subsection{Indicios de posesión conceptual y garantías para la aplicación de la Máxima del Valor Aparente}

Dicho esto, veamos cuáles son los indicios que nos permiten determinar que un individuo que realiza aplicaciones incorrectas de un término general posee, no obstante, el concepto comunitario expresado por ese término:

(a) Aplicaciones demostrativas del término relevante. Un hablante no experto en el dominio de un concepto puede realizar malas aplicaciones puntuales del término que lo expresa. Esto ocurre con Bert, quien dice, señalando su muslo, «esta artritis me está matando de dolor». Sin embargo, este mismo individuo, según nos dice Burge (1979: 104), sabe que sufre artritis desde hace años, por lo que también será capaz de realizar aplicaciones demostrativas correctas, ya que dirá, con verdad, cosas como "he sufrido artritis en esta rodilla». Lo mismo ocurre con Max. Este hablante considera, erróneamente, que todas las sillas, por definición, tienen patas y niega que el mueble sin patas destinado al asiento que ve en una tienda sea una silla. Sin embargo, también denomina correctamente «silla» a todas las sillas que hay en su casa, en casa de sus padres y de sus amigos, siendo todas ellas muebles con patas. No obstante, los casos de John y de Mary son distintos. El primero de estos hablantes considera que los orangutanes son un tipo de bebida de fruta que él afirma haber desayunado últimamente. Ante esto, no hará una aplicación correcta del término «orangután» cuando se le muestre un ejemplar de esta especie y se obstinará en aplicar el término al tipo de zumo de naranja, por ejemplo. Mary, por su parte, usará el término "creencia» para hablar de los melanomas y aplicará sistemáticamente esa expresión cuando se le muestren fotografías de esa clase de tumores.

(b) Apelación al grupo correcto de expertos dentro de la comunidad de hablantes. Bert acude a su médico, un experto en enfermedades, para comunicarle su temor de estar sufriendo artritis en el muslo. En este caso, el hablante acude al experto indicado, aunque ignore que sus temores con respecto a la artritis son 
infundados. Asimismo, Max, que no sabe que los asientos sin patas también se denominan «sillas», consultará al experto indicado (un decorador o el dependiente de una tienda de muebles) para cerciorarse de que sus usos del término «silla» son correctos. Ahora bien, ¿a quién acudirá John cuando quiera saber más sobre lo que él denomina «orangutanes»? Quizá al frutero, o a un botánico. En el caso de Mary, ocurriría algo parecido, ya que si este hablante delega en un experto el conocimiento de las condiciones de aplicación del término "creencia», dicho experto será, en su opinión, un médico, puesto que Mary considera que esa expresión es la denominación de un tipo de cáncer.

(c) Testimonio del hablante ante las rectificaciones. Supongamos, como pide Burge, que tras efectuar su consulta, el médico saca de su error a Bert. Tras esto resulta plausible que Bert admita que esa misma enfermedad que le afectaba a las articulaciones no es responsable de su dolor en el muslo, aunque él pensaba que sí. Esta posible declaración revela que su pretensión era referirse a/pensar en la artritis. Con respecto a Max, el hablante que considera que las sillas no pueden carecer de patas, ocurre algo semejante. Supongamos que un experto en sillas corrige a este individuo y le persuade de que los asientos que carecen de patas también se llaman «sillas». Tras esto, cabe pensar que Max dirá algo parecido a "pensé que las sillas sin patas no eran sillas», a partir de lo cual puede inferirse que, pese a su error, la pretensión de Max era pensar en las sillas cuando negaba que los asientos sin patas no podían denominarse haciendo uso del término «silla». Ahora bien, en el caso de John, este hablante no pretendía estar pensando en los orangutanes al decir «he desayunado orangutanes», sino que pensaba en el zumo de naranja pero denominándolo, por error o ignorancia, «orangután». Por este motivo, creemos que, tras la corrección relevante, un testimonio como «estaba equivocado al emplear la palabra 'orangután' cuando me refería a/pensaba en el zumo de naranja» es más plausible por parte de John que «me refería a/ pensaba en los orangutanes cuando me equivoqué al decir que ellos fueron $\mathrm{mi}$ desayuno en los últimos días». Algo similar ocurre en el caso de Mary. Este individuo denomina al melanoma "creencia», por lo que, en caso de que alguien le advirtiera de su error, parece extremadamente improbable que ella declare «antes de que me corrigieras, pensaba que las creencias se curaban con quimioterapia», más bien que «antes de que me corrigieras, cuando hablaba de creencias pretendía referirme al melanoma, que se cura con quimioterapia».

(d) Maximización del potencial explicativo de la atribución con respecto al comportamiento lingüistico y no lingüístico del sujeto. El fin de los informes psicológicos ordinarios sobre los pensamientos de terceros es integrar sus acciones y declara- 
ciones puntuales en una unidad coherente que nos permita explicar el comportamiento pasado del sujeto de atribución y prever su conducta futura. Si decimos, con Burge, "Bert cree que tiene artritis en el muslo», podremos volver inteligible su comportamiento en caso de que optara por auto-medicarse y doblar su ingesta de pastillas para la artritis. Asimismo, si Max hiciera un pedido de sillas a una tienda de muebles y decidiera devolver una de ellas porque carece de patas, mientras alega que él sólo quiere comprar sillas y ningún otro tipo de asiento distinto, sería máximamente explicativo decir que Max cree que todas las sillas tienen patas y, con ello, atribuirle el concepto de silla. Sin embargo, informar sobre John diciendo «él cree que debería volver a desayunar orangutanes» y que ése es el motivo por el que ha ido a la frutería sólo introduce confusión. Lo mismo ocurre con un informe tan falto de sentido como «Mary cree que ella nunca ha tenido ninguna creencia y que ese es el motivo por el que jamás ha visitado al oncólogo».

(e) Aprendizaje entroncado en la cadena comunitaria de transmisión históricocausal del uso del término. Generalizando las observaciones de Putnam sobre los términos de género natural en (1975b), cuando un hablante aprende el uso comunitario de un término «C» es frecuente que se utilicen definiciones ostensivas del mismo que involucran presentaciones de ejemplares paradigmáticos de C. El hecho de que las sillas paradigmáticas posean patas puede dar lugar a la formación de una creencia errónea por parte de Max, que generalizará las peculiaridades de esos ejemplares hasta creer que todas las sillas han de tener patas. Por su parte, cabe conjeturar que la creencia idiosincrásica de Bert sobre la artritis se debe a la cercanía del muslo con respecto a las rodillas, que, siguiendo las indicaciones de Burge, son articulaciones en las que Bert sufre artritis y, según cabe suponer, constituyen los «ejemplares» que han figurado en su aprendizaje del uso del término. No obstante, el aprendizaje de los términos «orangután» y «creencia» por parte de John y Mary no puede concebirse como una leve (y explicable) desviación de la cadena de transmisión comunitaria del término. Estos hablantes llevan a cabo aplicaciones del término que, por un lado, están radicalmente equivocadas con respecto a la extensión convencionalmente asociada al mismo pero que, por otro lado, siguen una pauta (idiosincrásica, eso sí), de tal manera que uno llama sistemáticamente "orangután» al zumo de naranja y el otro hablante denomina "creencia» al melanoma, de forma igualmente sistemática. Esto nos induce a pensar que la cadena de transmisión que sustenta el aprendizaje del uso de estos términos por parte de ambos individuos ha transcurrido por unos cauces distintos a los de la cadena de transmisión comunitaria, en el sentido de que ningún ejemplar que forme parte de la extensión convencional del término ha sido empleado en las definiciones ostensivas relevantes. 
Tras el examen de los cinco criterios expuestos, puede comprobarse que afirmar o rechazar que estos individuos posean un determinado concepto constituye en gran medida una expresión que se emplea desde el análisis filosófico para explicar que, a partir de indicios como los recogidos en (a)-(e), podemos confiar en que tales hablantes en contextos ordinarios están pensando lo mismo que, en principio, cualquier hablante del castellano pensaría usando las mismas palabras para expresarlo 7 . De este modo, los protagonistas de los casos «artritis» $\mathrm{y}$ «silla» son individuos semi-competentes en el uso de los conceptos de artritis y silla cuyas proferencias relevantes pueden interpretarse siguiendo la Máxima del Valor Aparente. Es más, debido al hecho de que ambos individuos confían en los expertos adecuados, realizan aplicaciones demostrativas del término mayoritariamente correctas, etc., lo más caritativo, en su caso, es atribuir a dichos hablantes la posesión del concepto comunitario. No ocurre lo mismo, sin embargo, en los casos «orangután» y «creencia», protagonizados por individuos manifiestamente incompetentes en el uso convencional de esos términos. Por tanto, las afirmaciones de que John tiene creencias sobre los orangutanes cuando hace uso del término «orangután» o de que Mary tiene creencias sobre las creencias cuando usa ese término suponen violentar de manera patente las intenciones lingüísticas (idiosincrásicas, eso sí) de ambos hablantes.

\section{Atribuciones y posesión conceptual en el seno de un proceso interpretativo}

En esta sección me ocuparé de continuar la tarea de Burge y explicar su dictamen sobre el protagonista del «caso orangután». Como vimos, dicho dictamen es doble; por un lado, para Burge resulta correcto informar acerca de los pensamientos de este individuo diciendo que «John cree que los orangutanes son bebidas de fruta» pero, por otro lado, no es apropiado decir "John cree que ha desayunado orangutanes las últimas semanas». Aunque no puedo asegurar que el propio Burge acepte todos los términos de mi explicación, considero que es

\footnotetext{
7 Asimismo, puede comprobarse que estos indicios se encuentran generalmente interrelacionados, en el sentido de que si un individuo sabe consultar al experto adecuado, categorizará de manera correcta la extensión de un concepto y, por tanto, será muy probable que sus creencias más básicas acerca de los ejemplares que componen dicha extensión sean verdaderas, aun cuando den lugar a malas aplicaciones puntuales del término, debido a su desconocimiento de cuestiones de detalle. Por este motivo, puede pensarse que los indicios (a)-(e), utilizados en sentido negativo, también tenderán a presentarse en bloque cuando nos encontremos con un individuo incompetente en relación a un determinado concepto.
} 
interesante aportar una serie de razones que pueden respaldar su diagnóstico, el cual, a mi modo de ver, goza de una intuitiva plausibilidad.

\subsection{El rompecabezas de Burge sobre el «caso orangután»}

Es preceptivo indicar, no obstante, que la doble opinión de Burge con respecto al individuo que protagoniza el «caso orangután» tiene un cierto aire paradójico. Así, por un lado, parece que muchas de las proferencias de John («voy a comprar orangutanes al supermercado", "acompaño mis desayunos de orangutanes exprimidos») no pueden interpretarse bajo la Máxima del Valor Aparente, ya que inducirían a error a los destinatarios del informe. Según he argüido en la sección anterior, este diagnóstico es certero en la medida en que John es un individuo radicalmente incompetente en el uso del término «orangután», que no posee el concepto de orangután ni piensa en los orangutanes al usar ese término.

Sin embargo, por otro lado, Burge sí admite que al menos una atribución, la realizada en el informe "John cree que los orangutanes son un tipo de bebida de fruta», es correcta y admisible, en contra de lo que opinaría la «tradición filosófica». Ahora bien, esta afirmación no encaja bien con uno de los indicios que Burge proporcionaba acerca de la competencia conceptual mínima, a saber, que la aptitud para recibir atribuciones en las que el término «C» figura en posición oblicua supone que dicho individuo posee el concepto $C$ relevante. Al mantener que la atribución "John cree que los orangutanes son un tipo de bebida de fruta» es correcta, estamos manteniendo, según las instrucciones de Burge, que John puede recibir una atribución en la que el término «orangután» figura en posición oblicua y que, por tanto, posee el concepto de orangután aun cuando sea semi-competente en su uso. Sin embargo, es evidente que esta afirmación no puede conjugarse con lo enunciado en primer lugar, a saber, que John no es competente en absoluto en el uso del término «orangután» y que no posee el concepto relevante.

\subsection{Presentación de la noción de atribución canceladora}

Para resolver esta situación, comenzaré distinguiendo dos tipos de atribuciones de contenidos. El primero de ellos contendrá aquellas atribuciones que, según los indicios que extrajimos en la sección anterior, consideraríamos incorrectas, en el sentido de que se han realizado aplicando la Máxima del Valor Aparente 
a individuos que usan un término general sin cumplir ninguno de los indicios (a)-(e) en relación a dicho término. El segundo tipo de atribuciones contiene informes que, aun cuando se realizan a los mismos individuos que son sujeto de las atribuciones del primer tipo y contienen en posición oblicua el término que ellos aplican de manera idiosincrásica, son informes «correctos» según las declaraciones de Burge:

Atribuciones tipo (A)

(3) «Amy cree que en esa tienda de electrodomésticos puedes encontrar elefantes de bajo consumo»

(4) «Eliott cree que la querella que le han comprado por su cumpleaños está rota»

(5) «Judy cree que su primo acostumbra a nadar en la puerta»

(6) «Kurt cree que Lara debería sembrar calefactores en su jardín» Atribuciones tipo (B)

(7) «Amy cree que los elefantes son un tipo de bombillas»

(8) «Eliott cree que las querellas son un tipo de cafeteras»

(9) «Judy cree que las puertas son un tipo de instalaciones deportivas»

(10) «Kurt cree que los calefactores son un tipo de plantas»

Establecidos estos dos grupos, puede comprobarse que los informes contenidos en el segundo grupo son aquellos que «caracterizan el error de los hablantes», como dice Burge, en el sentido de que hacen explícita la clase de equivoco o ignorancia radical que afecta a estos sujetos. Tales informes explican por qué estos individuos realizan aplicaciones idiosincrásicas de términos como «calefactor»o "puerta», aplicaciones que darán lugar a declaraciones tan carentes de sentido a primera vista como «me gusta el olor de los calefactores recién plantados», por parte de Kurt, o «debes ponerte un gorro cuando nades en una puerta climatizada», por parte de Judy. 
Pues bien, respaldamos la opinión de Burge en torno a la clase de atribuciones contenidas en el grupo (B), en el sentido de que no sólo parecen correctas —o más correctas que las de tipo (A) — sino que, precisamente por serlo, arrojan luz sobre la clase de incorrección que afecta a las atribuciones de tipo A. La explicación que ofreceremos acerca de este fenómeno es la siguiente. Denominaremos a las atribuciones de tipo (B) «atribuciones canceladoras», distinguiéndolas de las atribuciones contenidas en el grupo (A) porque, en primer lugar, ponen en evidencia el error o ignorancia radicales que afectan a un individuo con respecto al uso de un término y porque, en segundo lugar, poseen un rol distintivo en el proceso de interpretación de la conducta de los agentes, ya que desde el mismo momento en que puede establecerse la correción de una atribución de esta clase habrá de cancelarse o ponerse en suspenso la aplicación de la Máxima del Valor Aparente para aquellas proferencias del sujeto de atribución que contengan el término que concierne al error o ignorancia. En tercer lugar, cancelar la aplicación de esta Máxima supone que, a pesar de que las atribuciones canceladoras contienen el término «C» en posición oblicua, establecer una de estas atribuciones no implica que el sujeto acerca del cual versa el informe posea el concepto comunitario $C$ relevante.

\subsection{El rol de las atribuciones canceladoras en los procesos de interpretación}

Una vez presentada la noción de atribución canceladora, lo más conveniente es explicar sus propiedades y esclarecer dónde radica nuestra intuición de que son atribuciones correctas. Así, hemos dicho que el primer rasgo de estos informes es que revelan el error que subyace a las aplicaciones completamente incorrectas de los términos relevantes. Desde luego, debemos señalar que no hay una única forma de explicitar este error en relación a los hablantes que protagonizan nuestros ejemplos. En este sentido, en vez de la atribución «Amy cree que los elefantes son un tipo de bombillas» podríamos haber utilizado el informe "Amy cree que los elefantes son un tipo de lámparas incandescentes». Debido a esto, cabe sostener que no hay una única atribución canceladora en cada caso de incompetencia, sino que calificar de "canceladora» a una atribución depende de que ésta posea las propiedades relevantes, siendo la primera de ellas, como decimos, que haga explícita la clase de equívoco que afecta al sujeto de la atribución en relación a un término. Si esto es así, tanto «Amy cree que los elefantes son un tipo de bombillas» como "Amy cree que los elefantes son un tipo de lámparas incandescentes» son atribuciones que explicarían a un usuario competente en los 
términos involucrados en los informes por qué Amy hace un uso radicalmente incorrecto del término «elefante».

Por otro lado, en la recapitulación de atribuciones tipo (B) que hemos ofrecido utilizamos la forma del ejemplo que Burge emplea en su formulación del caso orangután ("John cree que los orangutanes son un tipo de bebida de fruta») debido a que esta clase de atribuciones resultan especialmente útiles a la hora de poner en evidencia que un individuo está aplicando de manera incorrecta un término al categorizar erróneamente su extensión, pensando, en definitiva, que un tipo de cosas (las que él denomina "C») son otro tipo de cosas distinto. No obstante, alguien podría objetar que también pueden elaborarse esta clase de atribuciones con respecto a Bert y Max, de quienes diríamos, respectivamente, que «Bert cree que la artritis es un tipo de enfermedad que afecta a los músculos» $\mathrm{y}$ «Max cree que las sillas son un tipo de muebles con patas». La respuesta a esto es que el estatus de una atribución en tanto que atribución canceladora depende de que dicha atribución revele un equívoco total con respecto a la extensión y uso de un término general, algo que no es el caso con respecto a Bert y Max, quienes creen, correctamente, que la artritis es un tipo de enfermedad y que las sillas son un tipo de muebles, esto es, saben en qué categoría de individuos ha de clasificarse la extensión de los términos «artritis» y «silla». En cualquier caso, se comprueba que para calificar a un equívoco de radical o relativo lo más conveniente es servirnos de los indicios de posesión conceptual como los señalados en la sección anterior. De este modo, detectar que un hablante realiza aplicaciones anómalas de un término general «T» y explicar sus errores con una atribución del tipo «S cree que los $\mathrm{T}$ son un tipo de $\mathrm{Z}$ con tales y cuales propiedades», no implica por sí solo que esa atribución sea una atribución canceladora, aunque la oración contenida en la cláusula-que de este informe no sea estrictamente verdadera. Para ello habría que evaluar qué grado de competencia tiene este hablante con respecto al uso del término " $\mathrm{T}$ » $\mathrm{y}$, en la misma medida, qué grado de inexactitud en la clasificación de la extensión del término por parte del hablante se revela en la cláusula-que de la atribución.

En segundo lugar, hemos observado que las atribuciones canceladoras tienen un papel muy definido en el proceso de interpretación de las proferencias de los hablantes. Empleamos la noción de proceso de interpretación para poner de manifiesto que los informes de contenidos mediante los cuales intentamos volver inteligible la conducta verbal y no verbal de los individuos son elaborados en una actividad continua y, por ello, planteados más o menos tentativamente en un ejercicio que se extiende en el tiempo. En este sentido, cuando desde la filo- 
sofía se toman en consideración ejemplos de informes mentales para evaluar su corrección o esclarecer sus presupuestos no deberíamos olvidarnos de que dichos informes se generan en el desempeño de una actividad, esto es, en el proceso de interpretación señalado ${ }^{8}$.

Es plausible pensar que en uno de estos procesos, cuando atendemos, por ejemplo, al uso que hace Amy del término «bombilla», las primeras proferencias en las que figura dicho término emitidas por este hablante serán aquellas que, interpretadas siguiendo la Máxima del Valor Aparente, darían lugar a informes de tipo (A). Para justificar esto, reparemos en que usualmente un hablante emplea un término general en las descripciones del desempeńo de sus quehaceres presentes y propósitos futuros, por lo cual, por ejemplo, lo aplicará para señalar una bombilla rota diciendo que «se ha averiado el elefante» o dirá «la comunidad de vecinos me ha encargado comprar elefantes adecuados para las luces del portal». Desde luego, aun cuando la Máxima del Valor Aparente constituya un presupuesto de los procesos de interpretación cuya aplicación resulta más inmediata («en tanto que hablante del castellano, este individuo quiere decir con sus palabras lo mismo que diría cualquier hablante de ese lenguaje»), una incompetencia tan manifiesta como la de Amy con respecto al término «elefante» prevendría a cualquier intérprete que tome a este individuo como un ser racional de realizar una aplicación automática de la Máxima.

${ }^{8}$ Con esto, acepto el planteamiento de Siebel (2005), quien advierte de que una aplicación simple del «Principio de Conceptualización" da lugar a ciertos puzzles sobre las actitudes proposicionales de individuos incompetentes. Dicho principio afirma que si un hablante $S$ amerita una atribución en la que figura un término general en la cláusula-que, entonces $S$ posee el concepto expresado por dicho término. Según este autor, sólo podremos decir esto último de $S$, si se exige, además, que $S$ tenga una concepción apropiada de los T, que sepa lo que es ser un T. Desde mi punto de vista, la integración de las atribuciones psicológicas en un proceso de interpretación supone adquirir cierta perspectiva desde la cual resulta plausible que no puede hacerse un uso irrestricto de dicho Principio, ya que, al menos, deben estar exentas el tipo de atribuciones que calificamos de canceladoras. Debe admitirse, no obstante, que en el seno del externismo psicológico ha existido una cierta tendencia literalista que contempla con simpatía dicho Principio. Cabe pensar que esto es fruto de la necesidad de defender el minimalismo en la competencia conceptual y de hacer énfasis en que un individuo puede poseer un concepto aunque no domine plenamente el uso del término relevante. Ahora bien, precisamente porque considero que el externismo es una tesis correcta y que también lo es el minimalismo en la competencia conceptual, los partidarios del externismo deberíamos esforzarnos en no acabar abogando por una tesis tan poco aceptable como la exigencia de una competencia completa para la posesión conceptual; nos referimos a la tesis de que basta con que un individuo use un término general de manera sintácticamente correcta en oraciones asertivas para que dicho individuo posea el concepto relevante y las atribuciones realizadas a partir de esos usos resulten apropiadas y explicativas. 
En este punto es donde el rol de las atribuciones canceladoras se revela con mayor claridad. Un hablante que intente dotar de sentido el comportamiento de Amy, alertado por el uso tan anómalo que este hablante realiza del término «elefante», podría emprender un cuestionamiento directo y preguntarle «¿qué entiendes por 'elefante'?». O, en caso de que Amy tuviera dificultades para explicarse o careciera en su vocabulario de un término como «bombilla»o «lámpara incandescente» que, según la interpretación convencional, serían sinónimos en su idiolecto de «elefante», el intérprete podría elaborar por sus propios medios un informe de tipo (B) debido a la persistencia de aplicaciones del término «elefante» por parte de Amy ante la presentación demostrativa de bombillas y su negativa a aplicarlo a los elefantes. Ahora bien, calificar de «atribución canceladora correcta» a uno de estos informes acerca de un hablante que no cumple en relación a un término dado ninguno de los requisitos de competencia (a)-(e) especificados, implica que en lo sucesivo la Máxima del Valor Aparente ha de cancelarse con respecto a todas las proferencias del hablante que involucran ese término hasta que se subsane el error o la ignorancia relevantes. En este sentido, creer correcto el informe "Amy cree que los elefantes son un tipo de bombillas» no supone atribuir a ese individuo el concepto en cuestión, porque justamente el papel de esa atribución es explicar a un tercero bien informado que este individuo no puede estar pensando en elefantes cuando profiere oraciones como «voy a comprar elefantes de 60w». De ser así, resulta preceptivo debilitar la afirmación de Burge de que un individuo será al menos mínimamente competente en un término (y poseerá el concepto relevante) si recibe atribuciones en la que ese término figura en posición oblicua que calificaríamos como correctas. Al contrario, parece que ciertas atribuciones, las que poseen un rol cancelador, pueden realizarse correctamente sin implicar posesión conceptual, debido al hecho de que se realizan para informar de la clase de error o ignorancia total que concierne al uso de un término por parte de un individuo. Con otras palabras, precisamente porque esta clase de atribuciones hacen explícita la razón de que un hablante sea radicalmente incompetente con respecto al uso un término general, dichas atribuciones no constituyen una señal de la posesión del concepto por parte del individuo relevante, sino de su carencia.

En este mismo sentido, puede reforzarse la idea de que la corrección de las atribuciones canceladoras no implica posesión conceptual (por parte del sujeto acerca del cual se informa) a partir del hecho de que los informes que hemos distinguido como tipo (B) podrían desempeñar dicho papel si fueran formulados mediante una descripción metalingüística del equívoco que sufre el agente con 
respecto al término relevante. Tomemos en consideración en este sentido los siguientes ejemplos:

(11) «Amy cree que la palabra "elefante" se usa para hablar de un tipo de bombillas»

(12) «Eliott cree que un tipo de cafeteras se llaman "querellas"»

(13) «Judy cree que debe usar el término 'piscina' para referirse al tipo de instalaciones deportivas en el que nada su primo»

(14) «Kurt cree erróneamente que ese tipo de planta se denomina "calefactor”»

Como puede comprobarse cada uno de estos informes puede clasificarse perfectamente como tipo (B) en la medida en que todos ellos cumplen los requisitos de las atribuciones canceladoras, a saber, explican el equívoco radical relevante, conllevan la suspensión de la aplicación de la Máxima del Valor Aparente y, de manera aún más clara que las atribuciones como (7)-(10), no comprometen al intérprete con que el sujeto que recibe la atribución posea los conceptos involucrados en dichos contenidos. Lo que se consigue en un proceso de interpretación mediante los informes (7)-(10) y (11)-(14) es exactamente lo mismo, a saber, mostrar que el uso de un término por parte del sujeto que recibe las atribuciones es completamente erróneo y, por tanto, que el contenido semánticamente expresado por aquellas de sus proferencias que darían lugar a informes de tipo (A) (interpretadas por el significado comunitario de los términos involucrados en ellas) y el contenido que este hablante quiere expresar son contenidos diferentes.

Asimismo, nuestro diagnóstico acerca de la diferencia entre las atribuciones (A) y (B) viene respaldado por el uso ordinario de otro tipo de informes sobre las declaraciones verbales de los hablantes. En ocasiones, cuando un hablante sufre un equívoco tan profundo y evidente como los protagonistas de los ejemplos que estamos planteando o cuando el desarrollo del proceso de interpretación no está lo suficientemente avanzado como para permitirnos determinar que un hablante no es competente en relación a un determinado concepto pero se sospecha esa falta de dominio, es usual informar de sus declaraciones empleando la expresión «dice que» para evitar comprometernos con que el hablante crea realmente lo que está diciendo. A este respecto, una vez que se ha obtenido un informe cancelador sería perfectamente natural, por ejemplo, aportar sentido a la conducta de Amy usando una oración como "Amy cree que los elefantes son un tipo de bombillas 
por eso ha dicho que en esa tienda de electrodomésticos puedes encontrar elefantes de bajo consumo». El informe cancelador es el fulcro en el que se apoyan el intérprete y el destinatario del informe acerca del comportamiento verbal y no verbal de Amy para explicar desde la perspectiva de la tercera persona qué clase de error o ignorancia afecta a este hablante en relación a un término, aportando una regla para traducir en el idiolecto del hablante los usos que ha hecho del término «elefante».

En resumidas cuentas, el hecho de que tanto en los informes tipo (A) como en los informes tipo (B) figure el operador « $S$ cree que» no consigue ocultar las diferencias que existen entre ambos, diferencias que se ponen de manifiesto cuando contextualizamos debidamente la elaboración de estos informes en un proceso de interpretación. Que pueda decirse puntualmente de un individuo $S$ que «S cree que $P$ » no implica eo ipso que $S$ posea ni que sea mínimamente competente en los conceptos involucrados en el contenido de que $\mathrm{P}$, especialmente si pretendemos que la paráfrasis "poseer un concepto» signifique algo o que, al menos, constituya un signo de que $S$ posee unas mínimas habilidades (normativamente sancionadas) en relación a los términos que expresan tales conceptos.

\section{Conclusiones}

Mediante la presentación de unos requisitos mínimos para la posesión conceptual y de la noción de atribución canceladora hemos intentado esclarecer el diagnóstico de Burge sobre la incorrección del informe "John cree que ha desayunado orangutanes» y la corrección del informe "John cree que los orangutanes son un tipo de fruta». Desde el momento en que integramos la elaboración de informes de contenidos dentro de un proceso de interpretación de las acciones y declaraciones verbales de los agentes, podemos comprobar que existen indicios objetivos de que un hablante no posee el concepto (comunitario) $C$ a pesar de que usa el término «C» relevante. Infiriendo por su comportamiento lingüístico y no lingüístico un informe cancelador que revele la clase de error del que es víctima el hablante, podemos confirmar que, en su caso, una aplicación de la Máxima del Valor Aparente con respecto al término «C» constituiría un claro atentado contra el Principio de Caridad. John no cree que los orangutanes recién exprimidos estén deliciosos ni Mary cree que jamás tuvo ninguna creencia. Una teoría de las atribuciones de contenidos individuados de manera externista debería ser capaz de explicar que estos individuos son lingüísticamente ignorantes pero no irracionales. 


\section{Bibliografía}

Bilgrami, A. (1992a). Belief and Meaning: the Unity and Locality of Mental. Cambridge, Blackwell.

- (1992b). "Can Externalism be Reconciled with Self-Knowledge?» Reimpreso en A. Pessin y S. Goldberg (eds.), The Twin Earth Chronicles: Twenty Years of Reflection on Hilary Putnam's "The Meaning of "Meaning" ". Londres, Sharpe, pp. 362-393.

- (2003). «A Trilemma for Redeployment», en Philosophical Issues, Vol. 13, No. 1, pp. $22-30$.

Boghossian, P. (1994). "The Transparency of Mental Content», en Philosophical Perspectives, Vol. 8, pp. 33-50.

Burge, T. (1979). "Individualism and the Mental». Reimpreso en T. Burge, Foundations of Mind. Nueva York, Oxford University Press, pp. 100-180.

- (1986). «Intellectual Norms and Foundations of Mind». Reimpreso en T. Burge, Foundations of Mind. Nueva York, Oxford University Press, pp. 254-274.

Davidson, D. (1997). "Indeterminism and Antirealism». Reimpreso en D. Davidson, Subjective, Intersubjective, Objective. Oxford, Oxford University Press, pp. 69-84.

Eвbs, G. (1996). «Can We Take Our Words at Face Value?», en Philosophy and Phenomenological Research, Vol. 56, No. 3, pp. 499-530.

Greenberg, M. (2007). «Incomplete Understanding, Deference and the Content of Thought». En UCLA School of Law Research Paper 07-30. Disponible en la dirección http://ssrn.com/abstract=1030144 y http://dx.doi.org/10.2139/ssrn.1030144, a cuya paginación remito [Acceso el 19 de junio de 2012].

Jackmann, H. (2000). "Deference and Self-Knowledge», en Southwest Philosophy Review, Vol. 16, pp. 171-180.

- (2003). "Charity, Self-Interpretation, and Belief», en Journal of Philosophical Research, Vol. 28, pp. 143-168.

Kripke, S. (1980). Naming and Necessity. Oxford, Blackwell.

- (1982). Wittgenstein on Rules and Private Language. Cambridge, Mass, Harvard University Press.

Nordby, H. (2004). «Incorrect Understanding and Concept Possession», en Philosophical Explorations, Vol. 7, No. 1, pp. 55-70.

Putnam, H. (1973). «Explanation and Reference». Reimpreso en H. Putnam, Mind, Language and Reality. Cambridge, Cambridge University Press, pp. 196-214. 
— (1975b). "The Meaning of "Meaning"». Reimpreso en A. Pessin y S. Goldberg (eds.), The Twin Earth Chronicles: Twenty Years of Reflection on Hilary Putnam's "The Meaning of "Meaning". Londres, Sharpe, pp. 3-52.

Russell, G. (2010). «Analyticity in Externalist Languages», en S. Sawyer (ed.), New Waves in Philosophy of Language, Londres, MacMillan, pp. 186-205.

Siebel, M. (2005). «A Puzzle About Concept Possession», en Grazer Philosophische Studien, Vol. 68, pp. 1-22.

Recibido: 28/06/2012

Aceptado: 17/10/2012 